\title{
Experiences of young adolescent mothers regarding adolescent motherhood in Oshana region
}

\author{
Sabina A. David ${ }^{1}$, Agnes Van Dyk ${ }^{2}$, Daniel Opotamutale Ashipala *2 \\ ${ }^{1}$ Department of Midwifery Nursing Sciences, School of Nursing, Faculty of Health Sciences, University of Namibia, Namibia \\ ${ }^{2}$ Department of General Nursing Sciences, School of Nursing, Faculty of Health Sciences, University of Namibia, Namibia
}

Received: May 21, 2017

DOI: $10.5430 /$ jnep.v7n12p39
Accepted: July 6, 2017

Online Published: July 17, 2017

\begin{abstract}
Background and objective: Adolescence is generally understood as a period of growing to maturity, transition from childhood to adulthood with physical and mental maturity. Nowadays girls enter puberty as young as 9 years which pose a health risk to adolescent pregnancy and early motherhood. The purpose of this study was to explore and describe the experiences of adolescent mothers as regards motherhood in Oshana region.

Methods: A qualitative, explorative, descriptive and contextual study was conducted among adolescent mothers in Oshana Region of Namibia regards their experiences on adolescent motherhood. Thematic analysis following Tesch's analysis process was employed.

Results: The findings of this study revealed that having a baby at a younger age have a negative effect on their future plans. It is an unfortunate fact that emerged from the study that many adolescent mothers lack the required form of support or social assistance both during pregnancy and in raising their children in the economically driven world of today. It is an unfortunate fact that emerged from the study that many adolescent mothers lack the required form of support or social assistance both during pregnancy and in raising their children in the economically driven world of today. It appeared that having a child gives an added impetus to their lives; and they felt that every decision or step they made would impact their babies' lives in one way or another. Conclusions: It appeared that having a child gives an added impetus to their lives; and they felt that every decision or step they made would impact their babies' lives in one way or another. They experienced distorted inter-personal relationships with families and friends related to motherhood as well as challenges on bringing up a baby and baby care. However adolescent mothers also had future ambitions on education, support, relationships and job attainment.
\end{abstract}

Key Words: Adolescence, Adolescent motherhood, Pregnancy, Motherhood, Experiences

\section{INTRODUCTION}

A recent estimate indicates that 16 million girls aged between 15-19 gave birth, wherein $95 \%$ of them occur in developing countries. ${ }^{[1]}$ Furthermore, $\mathrm{WHO}^{[2]}$ also indicated that the normative picture of adolescent mothers in Sub-Saharan Africa is that they look unhealthy with their babies, poorly educated, suffering from poverty, unemployed, shattered future and lack of access to reproductive health services. However, there will be some, though not in big numbers who survived the phenomenon of this adolescent motherhood.

Namibia, as a developing country is also not an exception. Adolescent motherhood is also a concern in Namibia. The increase in births from adolescents in Namibia is all over

\footnotetext{
* Correspondence: Daniel Opotamutale Ashipala; Email: dashipala@ unam.na; Address: School of Nursing, Faculty of Health Sciences, University of Namibia, Kaisosi Road, Rundu, Namibia.
} 
the country. Oshakati Intermediate Hospital birth records in Namibia, indicates that $9 \%$ births in January 2013 in were from young adolescents of $15-19$ yrs. $^{[3]}$ It is therefore quite noticeable according to the statistics mentioned earlier that adolescent motherhood is widespread in Namibia especially in rural areas of Oshana Region just like in other African countries.

Traditionally, Oshiwambo speaking people among which the study was conducted is that when a person got pregnant, she supposed to be supported until some months after giving birth when she can help herself. Motherhood was attained intentionally with the support of adults despite the age of that person. Unintended adolescent motherhood has now become prevalent and girls are delivering their first babies at a tender age in Namibia. "Not a girl, Not yet a woman, but already a mother". [4]

Furthermore adolescent motherhood is presented in the literature as today's challenge both nationally and internationally. It is regarded as a social health concern owing to its socioeconomic consequences. In this study adolescent motherhood means or referred to motherhood attained in a woman aged 15-19 years and adolescent is a term often used synonymously with teenager. When an adolescent girl delivers a baby, she becomes a mother which then form the basis of the family structure in which the child must grow up. It is then assumed that the adolescent's family structure should comply with the function of a family to enable her to provide the child with a safe, secure, nurturing, loving and supportive environment and to ensure that the baby have a happy healthy life.

\subsection{Problem statement}

The high incidence of adolescent mothers in rural areas is a matter of grave concern in Namibia, particularly in view of socio-economic of both the adolescent and the infant. When an adolescent gets pregnant it is usually unexpected, unplanned and a rude shock for her and the family. As she is a child herself, motherhood is believed to be problematic. On the other hand, in the past and traditionally, families provided support and guidance to new mothers. However the above support and guidance are disappearing in Namibian communities. It is assumed that adolescent mothers are thrown into an adult world without adequate preparation for parenting and in turn, may force them to make sacrifices and take decisions that they would never have thought of in their lives. Oshana region of Namibia is not an exception of adolescent motherhood. However, despite the high rate in adolescent pregnancies and motherhood, no data is documented on the experiences of these adolescent mothers and equally, little is said about the interventions in order to prepare, assist and support adolescent mothers during their motherhood period in Oshana Region. The following question arises: What are the experiences of adolescent mothers in the transition from adolescence to motherhood?

\subsection{Aim of the study}

The purpose of the study was to explore and describe the experiences of adolescent motherhood in Oshana region of Namibia.

\subsection{Research objectives}

The objectives were to explore and describe the experiences of adolescent mothers on motherhood in Oshana region of Namibia.

\section{STUDY DESIGN AND METHODS}

In this study, a qualitative, explorative, descriptive, contextual research design was used to explore and describe the experiences of adolescent mothers on motherhood.

\subsection{Population}

In this research study, the study population comprised of all first-time adolescent mothers aged 15-17 years that have experienced motherhood in Oshana Region of Namibia. The population includes all adolescent mothers who attended post-natal care at six weeks.

\subsection{Sampling and sample size}

In this study, purposive sampling was used to suitably select participants. In terms of a purposive sample participants are included in the study for the reason that they happen to meet the desired characteristics. The rationale behind choosing this sampling method is because it was considered the most appropriate method to address the purpose of the study and because it is useful for exploratory study. ${ }^{[5]}$ In qualitative research the sample size is not usually the focus as is the case in quantitative research. As this study is practice based, there was no specification of the sample size and data saturation determined sample size. ${ }^{[6]}$ This was achieved after fourteen (14) participants did not provide any different information from the previous informants.

\subsection{Data collection}

Researchers used in-depth interviews to collect the data. The interviews were tape recorded and transcribed verbatim with interviewee's permission. Audio- recording is an advantage because it helps the researcher to play back and listen were she failed to pick up something in writing. ${ }^{[7]}$

\subsection{Data analysis}

Data analysis was done by applying Tesch's method of open coding. ${ }^{[7]}$ This is a process of breaking down, examining, 
comparing and categorizing the raw data and at the end putting them together in a new way. ${ }^{[7]}$ This method was chosen because it is a systematic approach, and its procedures have been clearly described. The stages of data analysis were done based on the Tesch method of qualitative data analysis as proposed by Creswell. ${ }^{[7]}$ Step 1: Data transcription: Interviews were audio taped and subsequently transcribed verbatim. The following steps were employed namely: Researchers read through all of the transcripts from the interview data noted from observation and gathered as field notes, listened to the taped information on the cassette, carefully transcribed all taped interviews word for word, jotting down some ideas as they came to mind. It helped her to familiarize herself with the data and get a global understanding of the whole.

List of all topics was made and similar topics were clustered together to form themes. The researcher organized subthemes to see the development of themes.

The most descriptive wording for the topics was found and turned into subthemes. Subthemes were reduced by grouping topics together that related to each other.

Related subthemes were identified, grouped together and themes for each group were identified. Step 2: Data's reduction: After the data was transcribed, the interview transcripts were read through several times to obtain a sense and meaning of the whole.

Accordingly, researchers in this study used a frequency table to present the demographical data of the adolescent mothers who had participated in this study (see Table 1). A total of twenty mothers were identified for the interviews. Among those twenty mothers, four were between 18 and 19 years of age and, thus, not eligible to participate as they were not of the required age while two were not from the Oshana region. Accordingly, a total of 14 adolescent mothers only were interviewed. It is the richness of responses that is important and not the number of participants who share their experiences because the aim is to achieve, not quantity, but quality.

Table 1. Demographic data of the participants

\begin{tabular}{llll}
\hline $\begin{array}{l}\text { Characteristics } \\
\text { of participants }\end{array}$ & Categories & Frequency & Percentages \\
\hline Marital status & Unmarried & 14 & $100 \%$ \\
& Married & 0 & $0 \%$ \\
\multirow{2}{*}{ Educational } & Grade $1-5$ & 0 & $0 \%$ \\
level & Grade 6-10 & 13 & $93 \%$ \\
& Grade $11-12$ & 1 & $0.7 \%$ \\
Employment & Tertiary education & 0 & $0 \%$ \\
\multirow{2}{*}{ Age } & None & 14 & $100 \%$ \\
& 16 years & 8 & $57 \%$ \\
\hline
\end{tabular}

Published by Sciedu Press
The participants who were interviewed ranged in age from 16 to 17 years (mid adolescence) and they had all given birth to healthy new born babies. Not one of the participants was married and neither was in a steady relationship. All had reached the secondary level of education but not one had furthered her education or reached tertiary level either before or after giving birth. The majority of the participants had been in school at the time of conception. Furthermore, not one of them was employed during the period of the interviews. The language used/spoken mainly by the participants was Oshiwambo.

\subsection{Trustworthiness of the study}

To ensure trustworthiness of the transcribed data, the researcher kept transcripts and the audio taped interview information for referral. Transcriptions were sent to the study supervisor as per request to check whether all information was included in the study; this was done to prevent any information to be left out. Topics were identified and noted down in the transcriptions' document. Thereafter, topics were recorded in columns. Topics with the same meaning were linked together with lines. This resulted in the identification of sub-themes. These subthemes should be relevant for the research purpose. Throughout the analysis, the researcher attempted to gain a deeper understanding of registered nurses and student nurses' experiences. The findings were supported by verbatim quotes from the interviews, and they were sustained by relevant literature. A review of literature is conducted to generate a picture of what is known and not known about a particular situation. That information forms the background of the study. ${ }^{[8]}$

\subsection{Ethical consideration}

Permission was first of all granted by the University of Namibia's Research Committee by approving the proposed research study. Furthermore the approval was also requested and granted by the Ministry of Health and Social Services' Research Committee. In addition, approval was also obtained from the Oshana Regional Health Director, then written notifications were sent to the selected health facilities through the office of the Regional Health Director; and of course verbal agreements with the staff from facilities were the study was conducted.

Prior to the interviews, each participant was issued with a participant information sheet, explaining the aim of the study and requested their participation. A consent form was signed by all participants who agreed to participate in the study. Participants were given rights to withdraw from the study any time should they have wished to do so. Confidentiality and privacy were maintained throughout the interviews and 
focus group discussions. Anonymity was maintained in the reporting of the study findings. The tapes and transcripts were kept in a locked cupboard and data was entered in the computer and passwords were only known by the researcher

\section{RESULTS AND DISCUSSIONS}

Fifteen categories of coded data were identified and then linked together to form up themes. The emerging sub-themes were grouped together and combined into master themes as shown under the findings table. The table below indicate the themes and sub- themes emerged from the study.

Table 2. Themes and sub- themes that emerged from the experiences of adolescent mothers on motherhood in Oshana Region

\begin{tabular}{|c|c|}
\hline Themes & $\mathrm{S}$ \\
\hline $\begin{array}{l}\text { 3.1 Adolescent mothers } \\
\text { experienced various } \\
\text { feelings towards } \\
\text { motherhood }\end{array}$ & $\begin{array}{l}\text { 3.1.1 Fear of disclosing pregnancy, fear } \\
\text { of unknown and uncertainty } \\
\text { 3.1.2 Guilt, shame and embarrassment, } \\
\text { 3.1.3 Sense of confusion, unhappiness } \\
\text { and desperation } \\
\text { 3.1.4 Feelings of accomplishment }\end{array}$ \\
\hline $\begin{array}{l}\text { 3.2 Adolescent mothers } \\
\text { experienced challenges } \\
\text { related to motherhood }\end{array}$ & $\begin{array}{l}\text { 3.2.1 Health challenges (Physical and } \\
\text { psychological) } \\
\text { 3.2.2 Disruption in education or school } \\
\text { withdrawal } \\
\text { 3.2.3 Roles and lifestyle changes } \\
\text { Birth and becoming a mother }\end{array}$ \\
\hline $\begin{array}{l}\text { 3.3 Adolescent mothers } \\
\text { experienced lack of } \\
\text { effective inter personal } \\
\text { relationships with } \\
\text { families and friends }\end{array}$ & $\begin{array}{l}\text { 3.3.1 Loneliness, social isolation and } \\
\text { rejection by family/friends/boyfriends } \\
\text { 3.3.2 Lack of support and guidance by } \\
\text { family/friends/boyfriends and health } \\
\text { professionals }\end{array}$ \\
\hline $\begin{array}{l}\text { 3.4 Adolescent mothers } \\
\text { experienced burden on } \\
\text { caring and bringing up } \\
\text { babies }\end{array}$ & $\begin{array}{l}\text { 3.4.1 Lack of knowledge and skills on } \\
\text { routine care of the baby } \\
\text { 3.4.2 Financial difficulties and } \\
\text { dependency on other people's resources }\end{array}$ \\
\hline 3.5 Future ambitions & $\begin{array}{l}\text { 3.5.1 Support } \\
\text { 3.5.2 Relationship } \\
\text { 3.5.3 Job and educational aspirations }\end{array}$ \\
\hline
\end{tabular}

\subsection{Adolescent mothers experienced various feelings to- wards motherhood}

When a young adolescent becomes a mother, unplanned and not prepared, she will be affected physically, emotionally and socially because she did not expect to be in such a situation. The following themes and sub-themes have been aligned to the meaning units.

Adolescents thoughts that pregnancy was not going to happen to them, and have now to accept and cope with the new experience of motherhood, which is shocking. This is not what they wanted or what they chose. If motherhood is then attained or occurred early and unexpected by adolescents, they find themselves faced with many doubts and questions; which may result in stress which in turns brings about physical as well as emotional pathologies. These pathologies could create fear, shame, guilt feelings and embarrassment in adolescent mothers.

More often than not, they may have doubts and questions on their readiness to be parents and usually hide and fail to seek ante natal care. usually hide and fail to seek ante natal care.

"I was really afraid because getting pregnant was not in my mind. I could not believe it, though I did not use anything to prevent it. I was shocked." (mother 2)

\subsubsection{Fear of disclosing pregnancy, fear of unknown and uncertainty}

\section{Fear of disclosing pregnancy}

Adolescent mothers in this study experienced fear when they found out that they were pregnant especially in disclosing the pregnancy to the parents/guardians.

"I was really afraid because getting pregnant was not in my mind. I was shocked." (mother 6)

"I was scared to disclose my pregnancy because my family was not aware that I was engaged in sexual activities." (mother 1)

In his study of teenage pregnancy, Krefting ${ }^{[9]}$ also found that young mothers experienced difficulties in gathering enough courage to inform their parents and the immediate family. After confirming that they are pregnant it caused fear; anxiety, depression and anger which in turn prevent them to seek early ante-natal care.

\section{Fear of unknown and uncertainty}

Uncertainty and fear loom high among the adolescent mothers. Many have no idea how having a baby could have chaos of tough challenges with unforeseen uncertain prospects. Some believed in different ideas which were posed into their minds causing them to be more confused. Hearing and listening to stories from the community regarding pregnancy or birth was not an exception among these participants. They did not know what physical and emotional changes to expect and also what challenges during pregnancy and childbirth are awaiting them.

"People are saying that giving birth is not easy at all; it is between life and death. You are afraid to die, or your baby to die or both of you can die; but I did it miraculously." (mother 7)

An example is that in Oshiwambo culture, there are some articulations that reflect fear due to the believe that, if you 
are pregnant and your partner/husband sleeps with another woman and he comes early in the morning to find you in bed still asleep; then you will suffer a long painful labour or your baby or both of you may die. Some taboos prevail among some adolescent mothers who went to deliver at hospital.

"I was afraid to have long labour or my baby could have died because I stayed with my boyfriend and you are not sure whether he was seeing somebody else (girlfriend) and find you sleeping in the room (taboo). Luckily my labour went well; nothing happened or may be my boyfriend did not cheat on me." (mother 7)

In the area where the study was conducted, some taboos were believed by adolescents concerning the reduction of pain/contractions during the delivery process. Some people tend to believe what others are telling them, and only make a difference after going through themselves.

"I heard that if you wash your face with water the labour pains will subside, so I washed my face and hands while in hospital, but the pains did not change." (mother 10)

\subsubsection{Guilt, shame and embarrassment}

In this study some adolescents were emotional when they were asked how they felt when they found out that they were pregnant. They experienced guilt, shame and embarrassment, thus found it hard to disclose their pregnancies. Embarrassment was also from the situation of being denied the father of the baby. In Oshiwambo culture if a person is having a child without a father, the general conclusion is that the mother was loose and had many partners; and people have to wait until the birth of the baby for them to see whether the child looks like the accused father or not. These events were previously done in traditional courts. Even though, adolescent mothers still experience this kind of treatment in our communities.

"I went back to my mother and told her that the boyfriend is denying the pregnancy. She notified one of my uncles, and a meeting was called where we were all present and answer questions from my family members. I told them that he is responsible but he still denied the responsibility." (mother 3)

Since education is the key to success, it was also a reality in adolescent's mind though find themselves in pregnant situations. They also felt inadequate, incomplete to be responsible mothers.

"I was supposed to be in Grade 9 this year but now..." (mother 7)

Published by Sciedu Press
"I wish I have waited to finish school and get a job to enable me to support myself, my baby and my family." (mother 1)

However, some mothers of adolescent mothers though shocked at the beginning, showed their emotions by sympathizing and understanding towards their daughters and grand-daughters, while others shouted and sweared sometimes at them. In Oshiwambo culture, a parent to the adolescent mother feels hurt and uncomfortable among others if her daughter got pregnant and not married. The adolescent mothers have the following to say:

"My father, who did also not welcome the news, shouted at me and said that I must go and tell the one who impregnated me." (mother 1 )

"I was confused and afraid to tell my parents. I told myself to start with my mother who is closer to me, but it was also not easy at all. I felt so ashamed!" (mother 6)

Thomas $^{[10]}$ indicated also that pregnant adolescents experience feelings of guilt, anger and depression. He further stated that parents and families should be supportive and understanding to ensure that the pregnant adolescent adopts the lifestyle and changes due to pregnancy and birth.

While it is accepted in America for adolescents to freely disclose pregnancy, Namibia, specifically where the study was conducted has not developed that culture of freedom. More often than not, pregnant adolescents may have doubts and questions on their readiness to be parents and they usually hide their pregnancies and fail to seek for ante natal care as well as psychological care. These attitudes are having adverse effect on their health during pregnancy, and took long to reveal that they were pregnant.

"I use to hide the pregnancy and only revealed it after six months; I was scared and embarrassed to tell my auntie." ( mother 2)

Similarly adolescents in Soweto, South Africa reported that they suffered shame and embarrassment of having to reveal their pregnancies to the family or partners as well as peers. ${ }^{[11]}$ These researchers further commented on the silence of fear and shame that catch up families into inaction and the adolescent girls did not seek health care services if become pregnant.

Furthermore, in literature, a study conducted by Doctor Ziyane as reported by Mulongo ${ }^{[12]}$ in sense of confusion, unhappiness and desperation. 
The unexpected motherhood created disturbances in adolescent mothers and worse they did not think of getting pregnant though sexual active. In this study adolescent mothers suffered from confusion, unhappiness and desperation because they now have to accept and cope with the new responsibilities of motherhood.

\subsubsection{Confusion and desperation}

During motherhood an adolescent mother might feel disconnected from her dreams, goals and ambitions that were essential to her prior motherhood. She may experience confusion about what she wants to achieve in her life and what she want to do with her time that may leads to feelings of unhappiness. Some adolescent mothers felt hopeless when it comes to their perspectives of their future. They felt that their chances to go back to school are limited due to lack of support from family members and that no one will take care of their babies while at school. Some parents felt that when adolescents get pregnant early, it put an end to their life and have no way of improving. This evidenced:

"I have to forget about school because there will be no one to take care of my baby if I go back to school, said my mother." (mother 6)

Hughes and Ryals ${ }^{[13]}$ found the same in their study and quoted adolescent moms as raising problems of balancing school and being pregnant. It is not easy to balance school work with the care of the baby. They usually choose to take care of the baby and drop out of school. The demands of basic parenting make it difficult to keep up with school work. However, adolescent mothers as being the powerful predictor of the future constitute social problems in modern industrial societies.

"I don't know what to do! I feel like crying. Will I ever know what to do with the baby?" (mother 9)

Adolescent mothers were confused of what they wanted to achieve like continuing education with babies on their side. Both statements quoted indicated desperations and unhappiness since some have associated themselves with good careers after completing their education.

\section{Unhappiness}

The support for adolescent mothers during motherhood supposed to be from their mothers and close family members as well as from health professionals and from the society at large. Many factors that lead to unhappiness and discouragement are rooted in the society's expectations. Adolescent mothers blamed nurses and families who at times ignored their presence.
"Adolescent mothers need to be accepted and if possible allowed to go back to school, because these things just come unexpected." (mother 3)

Early motherhood is not what they wanted or what they chose, but when it is attained or occurred early and unexpected, they find themselves faced with many doubts and questions on their readiness to be parents and usually hide and fail to seek ante natal care. Failing to seek ante-natal care put them more to health risks simply because their bodies are still developing and may not cope with the pregnancy if not critically attended and supported by health professionals.

"I usually feel sad if I think of the support which I might not get." (mother 3)

"I don't know what to do. I feel like crying." (mother 8)

\subsubsection{Feeling of accomplishment}

Adolescent mothers revealed that some health professionals are doing their job well. They stated that they were informed about various health issues concerning pregnancy, labour and the baby care, which helped them. They were also given information on HIV and were tested that helped them to know their status.

"We both got HIV test and have negative status; I am very much happy about it and also that my boyfriend did not refuse to go for a test." (mother 1)

Though several participants were having fear, some participants were proud of giving birth and touching their babies for the first time was like a dream and they found it real and wonderful; they felt an immediate bonding when the baby was born.

"Nurses gave me my baby immediately after birth, for me to hold him for a while and asked me whether it is a girl or a boy. I was tired but I could hold him in my hands, that feeling...; I felt good and proud of myself." (mother 9)

This concurs with UNICEF ${ }^{[14]}$ some adolescent mothers who expressed their happiness and have not experienced difficulties either with pregnancy or delivery or any kind of assistance. They greeted their pregnancies with enthusiasm and joy. They felt blessed to have babies though young and promised to take care of them.

"Myself did not felt bad, I accepted that I got pregnant and was proud of it though I am too young." (mother 6) 
3.2 Adolescent mothers experienced challenges (physical and psychological challenges) related to motherhood

There are challenges associated with physiological immaturity in adolescents which includes condition like the disproportion of the fetal head to the mother's pelvic, hypertension, anemia etc. Adolescent mothers are at risks of motherhood challenges, physical as well as psychological, especially perinatal, as they tend to delay the seeking of ante-natal care. Risks like fetal death, infant mortality and morbidity as well as obstetric problems during and after giving birth are likely to occur. ${ }^{[14]}$

Adolescent mothers usually face health risks such as depression and other complications of pregnancy, birth problems and some other illnesses. Some physical changes that occur in adolescent mothers after birth are like depression, feeling of isolation, guilt, insomnia due to the care of the baby or due to the thinking of lack of support and many more. Lacking emotional and social support may also contribute to depression during pregnancy and after birth. ${ }^{[15]}$ Therefore adolescent mothers need love, comfort and a person close to them in order to develop self-esteem and self- reliance.

"I felt sad if I think of the support which I am likely not get, because the father of my child is denying the paternity." (mother 3)

\subsubsection{Health challenges}

In this study, physical and psychological challenges like the growing breasts, big abdomen, and other changes like mood swings, body changes and nausea were experienced.

"I have realized that I did not have my periods for one month and my breast got bigger." (mother 13)

"I was fine but I did experience nausea sometimes, but I did not vomit." (mother 9)

The woman's body is affected by pregnancy and due to the changes in the body, physical signs of pregnancy occur. The most affected organ is the uterus, which harbor the fetus throughout pregnancy. The other changes are like breast, menstruation and skin changes as well. These physical changes happen to enable the body maintaining pregnancy and also accommodate and nourish the fetus. The changes contribute also to the provision of strength and energy to the woman during the birth process. For adolescents, these physical changes are unexpected and some affect their lifestyle, Sellers ${ }^{[16]}$ has focused on adolescents not being emotional mature as yet to handle the pregnancy and its outcome. A study done by Ministry of Health and Social Published by Sciedu Press
Services (MOHSS) in Namibia has indicated that girls aged fifteen or younger are ten times more likely to succumb from pregnancy related complications than the older women of twenty to twenty nine of age. ${ }^{[17]}$

Some of the changes were evidenced:

"When I saw that I did not menstruate for a
month, I was a bit concerned and was worried
that, how come that I did not menstruate? Then
a second month passes too. I just went to the
clinic and get tested. It was found that I was
pregnant, I was in shock! All I thought was
'pregnant?"' (mother 7)

Menstruation is a periodic bleeding which women get every month as from puberty. The cycle can be then interrupted by pregnancy or any other factors which may hinder the menstrual flow to happen. This is a normal process which women are proud of. During adolescence, the body goes through changes and the responses to changes in body image can be a source of pride or on the contrary met with shame and embarrassment, specifically to adolescents who are still developing and have interest in their figures. They may feel embarrassed to be seen pregnant, which sometimes leads to hiding their pregnancies and not seeking ante- natal services on time.

"I use to hide the pregnancy and only revealed it after six months; I was scared and embarrassed to tell my auntie." (mother 2)

"I have realized that I did not have my periods for one month and my breast got bigger." (mother 13)

Meanwhile, nausea and vomiting also known as morning sickness, was also one of the complains by some participants in this study. This is the most frequent and troublesome symptoms during early pregnancy, and usually disappear before the 14th week of pregnancy. It occurs as a result of increased hormones (human gonadotrophin and estrogen) or due to increased utilization of glucose by the growing fetus. ${ }^{[18]}$ In addition, certain food my trigger vomiting especially food with a lot of sugar and this should be avoided. ${ }^{[19]}$ However, health professionals are knowledgeable in differentiating these conditions as long as women are seeking for assistance at health facilities.

"I was fine but I did experienced nausea sometimes but I did not vomit." (mother 9)

Despite these bad experiences, some adolescent mothers responded quite happily about these changes during pregnancy 
and express their wellness during the gestational period. Pregnancy affects all systems in the female body, including the nervous system which has to do with the mind; which is being triggered by many factors. These factors, which affect the behaviors of a woman, are sometimes attributed to hormonal alterations. This change marks the beginning of psychological tasks of pregnancy and the sense of mothering.

The adolescent mothers in this study experienced mood swings.

"When I felt not to be among people, I left and stay alone in the room, feel like crying." (mother

6)

Pregnancy is generally known as a time of psychological changes and as well as adjustments. According to Sellers, ${ }^{[16]}$ emotions, thoughts and behaviors go through alterations and continue until after the baby is born. Psychological changes especially in thoughts and attitudes of a pregnant woman may also bring about behavioral changes which could in turn leads to anxiety if there is no one to talk to or to support you.

"I usually feel sad if I think of the support I will need which I might not get." (mother 3)

However, these changes taking place are affected by social, cultural and the entire surroundings of the specific person involved, in this case adolescent mothers. It may have implications for one's whole life that may reflect on perceiving and adjustments to the pregnant situation.

\subsubsection{Disruption in education or school withdrawal}

Adolescent mothers face many obstacles in completing their high school education and one of those obstacles is adolescent pregnancy. It is a general believe that education is a way to success. The researcher in this study found out that adolescent mothers find it difficult to attend classes while pregnant or after giving birth. Some parents add to the weight that they cannot go to school until they give birth or until the baby is old enough to stay behind with families.

"My parents told me to stop going to school after I told them about the pregnancy. I should only go after giving birth and may be when the baby is big enough to stay behind with them, You see, when will that be." (mother 10)

Even though adolescent girls are being trapped to engage in unsafe sex, if pregnancy occurs, the consequences are not bearable at school and the shame may put them in a corner to stop attending. School environment will not be that conducive and consequently school withdrawal will result.

"You cannot go to school while pregnant, others

will laugh at you and sometimes the confrontation is from the teachers." (mother 2)
"I felt bad because pregnancy holds my school work behind and was ashamed to discontinue school." (mother 12)

The right to education for learners who are mothers is more strongly stated in Charter on the Right and Welfare of the African Child, to which Namibia is a signatory. Article (11) set measures for governments to be obliged to the realization of these educational rights, depending on the ability of a person. ${ }^{[20]}$ However, according to the adolescent mothers themselves, some parents as quoted previously in this study, still felt that girls who are pregnant should stop schooling and wait one year after giving birth On the other hand girls themselves felt embarrassed to be among others while pregnant.

"You cannot go to school while pregnant, others will laugh at you and sometimes the confrontation is from the teachers." (mother 2)

"I have to forget about school because there will be no one to take care of my baby if I go back to school, said my mother." (mother 6)

Nevertheless, this was not only from the educational point of view where adolescents got hard, but also some of the basic health services were hard to get. Inaccessibility to health services according to the adolescent mothers contributed also to the unexpected pregnancies because contraceptives were not easily reached when adolescent happens to seek for it. The access was not that easy for them as evidenced in this study.

"When you go to the clinic for contraceptives, the nurses will not attend to you because the clinic is always full of sick people. They attend first to the sick, so we went and came back unattended." (mother 5)

The researcher felt that if adolescent mothers are assisted and supported during their motherhood, they can courageously further their education and have a bright future. Collins' study ${ }^{[21]}$ is in accord with the idea that parenting classes for teen mothers can be very much helpful, to shape up their future.

\subsubsection{Roles and lifestyles changes}

The study found that adolescent mothers get it hard in changing to mothering roles. They were used to the freedom style but now the work seems doubled.

"To be a mother is hard, you are no longer having that freedom, and you are a mother now... So, there is no freedom to go out with friends when you feel like going." (mother 13) 
Giving birth is a natural process whereby a baby is pushed out through the birth canal ${ }^{[22]}$ describes childbirth as a transition period from childhood to parenthood and from childless to having a child. This brings up significant social and psychological role changes for the parent. The woman will be proud to confirm her biologic capability of reproducing through giving birth. However, this proudness can be distorted if there is fear and uncertainty or if the person is ill prepared to be a parent. Some participants were proud of giving birth and touching their babies for the first time was like a dream and they found it real and wonderful; they felt an immediate bonding when the baby was born. This was evidenced by the following statement:

"Nurses gave me my baby immediately after birth, for me to hold for a while and asked me whether a girl or a boy. I was tired but I could hold him in my hands, that feeling...; I felt good and proud of myself." (mother 1)

\subsection{Adolescent mothers experienced lack of effec- tive inter-personal relationships with families and friends}

Adolescent mothers in this study reflected on how having a baby at a younger age affected their future plans. Having a child seems to give them an added impetus in their lives; and felt that every decision or step they made will impact their babies' life in one way or another and should make sure that those decisions are good with a great support from their families and health workers.

However, those adolescents who seek for help at clinics before getting pregnant, seems not be satisfied with the assistance. After probing more on when visiting the health facilities for information and contraceptives, it seems that health workers doesn't make time to attend to them and came unattended.

"When you go to the clinic for contraceptives, the nurses will not attend to you because the clinic is always full with sick people. They attend to the sick first, we went and came back unattended." (mother 5)

\subsubsection{Loneliness, social isolation and, rejection by family, friends/boyfriends}

Some adolescent mothers in this study experienced feelings of loneliness, isolation and rejection as well as disconnection from families, friends including boyfriends or partners. Loneliness can lead to feelings isolation of unhappiness and affect daily life and cause disturbances in the adolescents' relationships with their friends and loved ones.
"Days seems the same, you do not have some other things to do. Is just a baby! I never see my friends, they won't even understand me if I think of calling them." (mother 7)

This statement is in line with the study done by Voeten ${ }^{[23]}$ in Okavango, Namibia that teenage pregnancy experienced psychological problems of loneliness, shame, fear and isolation. Adolescent mothers would no longer have the comfort and security of their peers with whom to socialize, because the other friends do not want to be associated with her anymore. However, having someone to talk to is important and can help a person to relieve stress or worries. This study revealed that some parents though not happy rejected their daughters and passed nasty comments because of the pregnancy.

"My father, who did also not welcome the news, shouted at me and said that I must go and tell the one who impregnated me." (mother 1 )

\section{Social isolation}

Adolescent mothers felt isolated in their homes where the interactions with friends diminished. They expressed their unpreparedness to take on motherhood demands, and this need guidance and support. Their youthful, romantic days before being a parent changed dramatically to serous maternal responsibilities of daily care of the newborn.

“...You have a responsibility of a baby especially when you are not working and the boyfriend is denying the responsibility (tears running over cheeks). You don't really know what to do." (mother 3)

They face responsibilities unlike those of their friends who don't have babies. An adolescent mother loses her freedom to go out all the time or hang out with friends. She has to line up child care if she want to socialize which can leave her isolated from friends and friends might also stop visiting and calling, because they feel they don't have much in common anymore.

"My friends don't visit me, is only if I go to them and chat on things which are not pregnant related to pregnancy." (mother 3 )

\subsubsection{Lack of support and guidance}

Having a baby confines the mother's social activities. It is an unfortunate fact in this study that many adolescent mothers lack and requires some form of support or social assistance during pregnancy and in raising their children in today's economically driven world. In most cases biological fathers of babies born to these adolescents are not committed to either 
a medium or long term relationship. If they are young obviously they also do not have economic security to support the mother and the child; and if they are old (sugar daddies) they probably have other relationship which they consider more important rather than to commit to an adolescent mother. Consequently, adolescent mothers are said to be getting no support system from outside their families and most often than not they experience poverty.

"I was lucky to have my grandmother, she assisted me nicely and luckily she is also a counselor." (Mother 7)

However, adolescent mothers in this study experienced boredom and loneliness, except taking care for the baby.

"Some days are really too boring and long, at night the baby cries. You will have sleepless night alone! Days seems the same, you do not have some other things to do. Is just a baby! I never see my friends, they won't even understand me if I think of calling them." (mother 4)

Lack of support may aggravate other problems in adolescent mothers such as attenuate education, underemployment and unstable relationship with father of child as well as health for themselves and their babies. ${ }^{[24]}$

In the absence of the support, adolescent mothers are struggling to grow up and form their identity, and then an added task of piloting motherhood. It is much essential for the adolescent mothers to get support, be it tangible, emotional or social support; however differing support networks ( family, friend, partners) are sometimes lacking or absent from their lives.

The most frequently providers of support to adolescent mothers are their mothers and grandmothers; and sometimes the fathers of their children. It was noticed in this study that adolescent mothers who live with their parents or relatives are more likely to return to school and wish to graduate, get employed and be economically independent.

"My family said they will look for somebody to look after my baby (baby sitter), for me to go back to school." (mother 4)

The thought of adolescents conceiving and giving birth to a baby is terrifying because the reality is that they will raise their children while they are still children themselves. Several researchers on adolescent pregnancy recommend that adolescent mothers are not prepared for parenting which is associated with lack of experience and what is required to raise a happy, healthy child.
"You are not sure about the choice and caring you are giving for your little one who cannot tells you her needs." (mother 5)

Adolescent mothers had different perceptions regarding newborn babies. Some felt that with the guidance from midwives they can manage while others felt the opposite of uneasiness. Midwives play major roles in facilitating mother-infant bonding as well as feeding of the baby. The condition of the baby should thus be assessed effectively in a comfortable environment, and any birth complications are intervened urgently for the babies to survive.

\subsection{Adolescent mother experienced burden of caring and bringing up a baby}

In this study adolescent mothers experienced physical and psychological challenges on baby care. The thought of adolescents conceiving and giving birth to a baby is terrifying because the reality is that they will raise their children while they are still children themselves. Several researchers on adolescent pregnancy recommend that adolescent mothers are not prepared for parenting which is associated with lack of experience and what is required to raise a happy, healthy child. In addition, Starks et al. (2008), ${ }^{[25]}$ further states that the majority of adolescent mothers lack patience to care for a child.

\subsubsection{Lack of knowledge and skills on routine care of the baby}

To adolescent mothers who often lack maturity and experience, knowledge and skills on baby care don't always come naturally. They might not know how to do the basics of baby care, which of course was expressed in the study. Educational programme was then a necessity for them to gain necessary skills for baby care. It was hard for these adolescent mothers to get used to handling, breastfeeding, bathing and dressing newborn babies as well as other routine care for a baby.

These were evidenced:

"The baby is too slippery; I am having fear when bathing may be to fall off my hands." (mother 6)

"The umbilical cord looks so fresh and scary, but you still need to clean it. Then it dried and fall off, then I was at least relieved." (mother 7)

Another aspect during this period after birth is the growing relationship of the mother and baby, which create a tie or bond between the two. Bonding is one of the most pleasurable aspects of baby care and should occur during the sensitive time in the first hours after giving birth depending on the 
condition of mother and baby. Physical closeness through putting the baby on the chest (skin-to-skin) while feeding and gently stroking him or her, can promote an emotional connection. For the baby, this attachment contributes to emotional growth which affects its physical growth as well. ${ }^{[16]}$

LaRocca $^{[26]}$ stated in her article on motherhood, how one couple got separated because of the inconsolably cry of their baby and the husband withdrawn and never helped, consequently she felt abandoned and resentful. The couple only reconcile after a professional help because the husband was hurt, frustrated and disappointed by the crying of their baby.

These expressions were quoted:

"My biggest problem I have had is when my baby cried almost the whole night; I got worried about it and I hardly slept but now she is perfectly fine." (mother 10)

"To be a mother is difficult, though you are blessed with a baby, and nicely fed, you need to take care of the baby and breastfeed even during the night. You won't sleep properly." (mother 14)

Despite challenges in some adolescent mothers on baby care, some were positive and face those challenges with courtesy. They felt that giving birth was a blessing and the arrival of their babies provides a deep sense of joy and accomplishment, thus expressed that they did not regret getting pregnant.

The responses were:

"Myself did not felt bad, I accepted that I got pregnant and was proud of it though I am too young. I believe that if you got pregnant, don't thing of abortion or other things if you are not getting support, just take it easy and continue with life. I felt that God has blessed me with the pregnancy and now the baby." (mother 7)

The aforementioned statement is giving a good picture of a considerate adolescent who regard some of the deeds commonly done in the country as evil actions. These are like criminal abortion, dumping and battering of children and sometimes-even killing of newborn babies which are nowadays' news almost every day. Namibia needs responsible parents, who will face challenges of being parents, and would like to have adolescent mothers who will meet life with optimism, confidence, competence and compassion, though very young.

"I will be supported in taking care of the baby.

My family will buy clothes and food for me and the baby." (mother 8)
Compared to older women, adolescent mothers are more impatient and punitive, and less nurturing. They may also be so stressed by the challenges of motherhood that they become depressed, develop poor self-esteem, and are not able to provide their children with emotional stability.

\subsubsection{Financial difficulties and dependency on other peo- ple's resources}

Adolescent mothers are faced with dual challenges of assuming the mother role and to bond with their babies, yet they also expect to be mothered and reassured by their mothers or grandmothers. Some adolescent mothers expressed their experiences of being assisted and supported by their mothers or grandmothers concerning the resources. The role of this support is critical however differing support were sometimes lacking during their motherhood period.

Though life can change dramatically in adolescent motherhood, having a baby should not end their dreams and goals for the future. In this study, adolescent mothers expressed their willingness to be supported especially emotional support which includes intimacy or closeness, guidance, encouragement and reassurance.

Adolescent mothers reflected on how having a baby at a younger age affected them and their plans for the future. Adolescents were forced to deal with developmental tasks while still young and mothers, often ill prepared, with few resources and stressed. In some ways they felt that they got matured through child birth and accepted the context they found themselves. They learned a lot from challenges on pregnancy as well as by giving birth and the taking care of the baby.

Adolescent mothers' perspectives on their future were quite positive as quoted:

"I lost my job as a baby sitter due to epilepsy and being pregnant, but now that I am on treatment and have my baby, I will look for a job in order to survive." (mother 8)

\subsection{Future ambitions}

Though life can change dramatically in adolescent motherhood, having a baby should not end their dreams and goals for the future. In this study, adolescent mothers expressed their willingness to be supported especially emotional support which includes intimacy or closeness, guidance, encouragement and reassurance.

Adolescent mothers reflected on how having a baby at a younger age affected them and their plans for the future. Adolescents were forced to deal with developmental tasks 
while still young and mothers, often ill prepared, with few resources and stressed. In some ways they felt that they got matured through child birth and accepted the context they found themselves. Having a child seems to give adolescent mothers an added impetus in their lives; and felt that every decision or step they make will impact their babies' life in one way or another and should make sure that those decisions are good with a great support from especially their families.

In this study some fathers if not all were also adolescents and they were unemployed or learners themselves and their support for basic necessity was limited that was provided mainly by their parents. The study revealed that support from fathers of babies, families as well as from health professionals was poor. This is what adolescent mothers said:

\subsubsection{Support}

Support from health professionals is regarded as a kind of care with specific knowledge and art and is a form of helping the adolescent mothers concerning motherhood. Orem is explaining care as to attend and serve others or to provide close care of a person, while support means to sustain in an effort in order to prevent failure, or avoiding unpleasant decisions or situations and also to do something without stress. The study revealed that support from fathers of babies, families as well as from health professionals was poor. This is what adolescent mothers said:

"Adolescent mothers needs guidance, support and assistance and to be accepted and if possible allowed to go back to school, because these things comes just unexpected." (mother 3 )

\subsubsection{Relationships}

Sometimes conflicts arise in the family because parents are not providing the support wanted by the adolescents or maybe they are not reaching consensus about family or social values. The adolescent mothers' perspectives on their future were quite positive because they felt their lives were not over due to early motherhood and positively looked ahead. They expressed their willingness for support especially emotional support which includes intimacy or closeness, guidance, encouragement and reassurance.

"Parents should be open with us and understand adolescents. They should honestly talk to us on issues pertaining to our development, reproductive health for example menstruation and pregnancies and sexual issues. Girls are only learning from their peers or through discovery learning." (mother 7)

\subsubsection{Education and job aspiration Educational aspirations}

Young mothers should be encouraged to take part in moth- erhood classes and to continue with their education by all means.

"I am going to continue with school because I have already passed grade 10 . I want to succeed and help my child and my other sisters." (mother 1)

\section{Job aspirations}

Adolescent mothers face challenges in obtaining jobs which meet their financial needs due to limited education.

“... Now that I am on treatment, I can look for

a job to survive." (mother 8)

\section{Conclusion}

It was clear from the findings that adolescent experienced emotions and feelings towards pregnancy and for being mothers like support and relationships. They experienced distorted inter-personal relationships with families and friends related to motherhood as well as challenges on bringing up a baby and baby care. However adolescent mothers also had future ambitions on education, support, relationships and job attainment. Furthermore, findings revealed that adolescent mothers are not receiving the support they need during motherhood therefore a support programme is needed to cater for challenges they are encountered during motherhood.

\subsection{Recommendations}

The following recommendations are made based on the findings of this study:

- The Ministry of Health and Social Services should provide technical support to the stakeholders for them to implement and extend the programme. Health professionals should give the necessary information and support to adolescent mothers through Adolescent Friendly Health Services.

- The Adolescent Friendly Health Services (AFHS) which was implemented by the Ministry of Health needs to be strengthened by health workers in each health facilities, because currently not all health facilities are adolescents user friendly and consequently includes the motherhood programme.

\subsection{Limitation of the study}

Participation was voluntarily and data were obtained from one region and one tribe, thus cultural homogeneity in the sample may prevail which may have an implication on the ability of generalizing the findings across the country.

\section{ACKNOWLEDGEMENTS}

We wish to thank all the respondents who took part in this 
study. The authors contributed as follows: Sabina Aisheoina David is the principal investigator, Agnes Van Dyk, supervised the project and Daniel Opotamutale Ashipala wrote the manuscript.

\section{CONFLicts OF InTEREST Disclosure}

The authors declare no conflicts of interest.

\section{REFERENCES}

[1] WHO. Adolescent pregnancy: a cultural complex issue. Bulletin, World Health Organisation. 2009; 87: 410-411. https://doi.or g/10.2471/BLT .09. 020609

[2] WHO. Adolescent pregnancy: unmet needs and undone deeds: a review of the literature and programmes. Issues in Adolescent Health and Development. (WHO discussion papers on adolescence). 2007.

[3] Ministry of Health and Social Services: Health Information System. Oshakati District HIS Office: Republic of Namibia. 2013.

[4] New Era Nov 9. Not a girl Not Yet a woman, But already a mother. Africa News Services articles from Nov. 2004.

[5] Burns N, Grove SK. The practice of Nursing Research, conductcritique and utilization. Philadelphia: WB Saunders. 2005.

[6] Polit DF, Hungler BP. Nursing research principles and methods.Philadelphia, PA: Lippincott. 2006.

[7] Creswell JW. Research design: Qualitative \& quantitative and mixed methods approaches 3 edition. Thousand Oaks, CA: Sage; 2009.

[8] Guba EG, Lincoln YS. Competing paradigms in qualitative research. London: Sage; 1994.

[9] Krefting L. Rigor in qualitative research. The assessment of trustworthiness. 1991

[10] Thomas R. A general inductive approach for qualitative data analysis. School of Population Health. University of Auckland, New Zealand. August 2003.

[11] Norris V, Ginsburg M. The silent truth of teenage pregnancies: Birth to twenty Cohort's next generation. South African Medical Journal. 2006; 96: 122-124. PMid:16541552

[12] Mulongo EMN. Young Single Motherhood contested notions of motherhood and sexuality in Policy discourses/Program interventions.Working Paper Series No 423. Institute of Social Studies. 2502 CT The Hague -The Netherlands. 2006.

[13] Hughes T, Ryals J. What pregnancy means at different ages. The Student Voice.University Academy Charter High School, Jersey City. 2011.

[14] UNICEF. Teenage motherhood in Latin America and the Caribbean: Trends, problems and challenges. Challenges Newsletter on Progress
Towards the Millennium Development Goals for a Child Rights Perspective. 2007.

[15] Kamara AR. The impact of teenage pregnancy and teenage motherhood on the lives of young girls. 2011. Available from: http://africayoungvoices.com/2011/10/theimpac t-of-teenage-pregnancy-and-teenage-moth....

[16] Sellers PM. Midwifery: A textbook and reference book for midwives in Southern Africa Vol. I. Cape Town: Juta. 2007.

[17] Ministry of Health and Social Services \& UNFPA \& UNICEF. Proceedings of the National Safe Motherhood Conference, held on 26-28 November 1992, Windhoek. 1992.

[18] Sellers PM. Midwifery: A textbook and reference book for midwives in Southern Africa Vol. I. Cape Town: Juta. 2007.

[19] Austin K. Coping with pregnancy morning sickness. 2006. Available from: http://www. ArticleGeek. com

[20] Hubbard D. Realising the right to education for all: School policy on learner pregnancy in Namibia. 2009. Available from: http: //www . kas.de/upload/auslandshomepages/namibia/children

[21] Collins Q. Cons to Parenting Classes for Teen Mothers to how Contribute. 2010. Available from: http://www. ehow.com/list-733 2768-cons-parenting-classes-teen-mothers.html

[22] Ladewig PW, London MC, Moberly SM, et al. Contemporary Maternal Newborn Nursing Care. (5th ed). New Jersey 07458: Pearson Educ. Inc. 2002.

[23] Voeten H. Teenage Pregnancy in Namibia: Problems, Causes and Policy Recommendations. University of Utrecht, Netherlands. 1995.

[24] Gee C, Rhodes J. Adolescent mothers' relationship with their children's biological fathers: Social support, social strain and relationship continuity. Journal of Family Psychology. 2003; 17: 370-383. PMid:14562461 https://doi.org/10.1037/0893-3200.17.3 .370

[25] Starks P. Teen mothers' experiences in the New Beginning Parent Education Programme. University of Central Oklahoma. 2008. Available from: http://www.grin.com/en/doc280723/teen-mot hers-experiences-in-the-new-beginning-p

[26] LaRocca L. The reality of motherhood: What nobody tells you.Grand Valley on Canada. From New Beginnings. 2006; 23(4): 148-212. 